\title{
Reproducing Kernel Method for Solving Nonlinear Differential-Difference Equations
}

\author{
Reza Mokhtari, Fereshteh Toutian Isfahani, \\ and Maryam Mohammadi
}

Department of Mathematical Sciences, Isfahan University of Technology, Isfahan 84156-83111, Iran

Correspondence should be addressed to Reza Mokhtari, mokhtari@cc.iut.ac.ir

Received 19 March 2012; Accepted 19 May 2012

Academic Editor: Ferhan M. Atici

Copyright (C) 2012 Reza Mokhtari et al. This is an open access article distributed under the Creative Commons Attribution License, which permits unrestricted use, distribution, and reproduction in any medium, provided the original work is properly cited.

On the basis of reproducing kernel Hilbert spaces theory, an iterative algorithm for solving some nonlinear differential-difference equations (NDDEs) is presented. The analytical solution is shown in a series form in a reproducing kernel space, and the approximate solution $u_{n, m}$ is constructed by truncating the series to $m$ terms. The convergence of $u_{n, m}$ to the analytical solution is also proved. Results obtained by the proposed method imply that it can be considered as a simple and accurate method for solving such differential-difference problems.

\section{Introduction}

Differential-difference equations play a crucial role in modelling of much physical phenomena such as particle vibrations in lattices, currents in electrical networks, pulses in biological chains, discretization in solid state, quantum physics, textile engineering, stratified hydrostatic flows, and so on. See, for example [1-14].

Recently, there have been lots of efforts in giving exact or approximate solutions of NDDEs. For instance, Zhu [4] extended the Exp-function method for solving NDDEs. In this method, exact solutions are sought in the form of an exponential type rational function in which both the numerator and denominator are polynomials of exponential functions. Mokhtari [5] applied the variational iteration method in which a correction functional is established by a general Lagrange multiplier, which can be identified optimally via the variational theory. The method gives rapidly convergent successive approximations of the exact solution. Qian et al. [6] extended the multilinear variable separation approach to a special differential-difference equation. Baldwin et al. [7] presented an algorithm to find exact travelling wave solutions of differential-difference equations in terms of the tanh function 
and found kink-type solutions in many spatially discrete nonlinear models. Wu et al. [8] and Wang et al. [9] extended the Adomian decomposition method to solve NDDEs. The technique is based on a decomposition of the solution of NDDE in a series of functions. Each term of the series is obtained from a polynomial generated from an expansion of an analytic function into a power series. Many authors [10-14] applied the homotopy perturbation method to solve NDDEs. The method, which is based on homotopy, constructs a continuous mapping of an initial guess through an auxiliary linear operator, and an auxiliary parameter is used to ensure the convergence of solution series.

The theory of reproducing kernels [15] was used for the first time at the beginning of the 20th century by S. Zaremba in his work on boundary value problems for harmonic and biharmonic functions. This theory has been successfully applied to fractal interpolation [16], solving ordinary differential equations [17-20] and partial differential equations [21, 22]. The book [23] provides excellent overviews of the existing reproducing kernel methods for solving various model problems such as integral and integrodifferential equations.

In this study, a general technique is proposed for solving some NDDEs in the reproducing kernel space. The main idea is to construct the reproducing kernel space satisfying the conditions for determining solution of the NDDEs. The analytical solution is represented in the form of series through the function value at the right side of the equation. For illustration, we apply this method to the Volterra lattice equation, the discretized mKdV lattice equation and the discrete sine-Gordon equation. The advantages of the approach lie in the following facts. The approximate solution $u_{n, m}(t)$ converges uniformly to the analytical solution $u_{n}(t)$. The method is mesh-free, easily implemented and capable in treating various boundary conditions. Since the method needs no time discretization, there is no matter, in which time the approximate solution is computed, from the both elapsed time and stability problem, point of views. Also we can evaluate the approximate solution $u_{n, m}(t)$ for fixed $m$ once and use it over and over.

In the next section we describe how to solve a NDDE through the reproducing kernel method and verify convergence of the approximate solution to the exact solution. Several numerical results are presented in Section 3. The last section is a brief conclusion.

\section{Construction of the Method}

Consider the following NDDE

$$
L u_{n}(t)=N\left(t, u_{n+p_{1}}, \ldots, u_{n+p_{d}}\right), \quad t \in(0, T]
$$

where $L$ is a linear differential operator of one-order derivative in $t, N$ is a nonlinear differential operator and $n, p_{i} \in \mathbb{Z}$. Since nonhomogeneous initial condition can be reduced readily to the homogeneous one, we only consider the following homogeneous initial condition

$$
u_{n}(0)=0 \text {. }
$$

We assume that (2.1)-(2.2) has a unique solution. 
Definition 2.1. $W[0, T]=\left\{u(t) \mid u(t), u^{\prime}(t)\right.$ are absolutely continuous in $[0, T], u^{\prime \prime}(t) \in L^{2}[0, T]$, $u(0)=0\}$. The inner product and norm in $W[0, T]$ are defined, respectively, by

$$
\begin{gathered}
\langle u, v\rangle_{W}=\sum_{i=0}^{1} u^{(i)}(0) v^{(i)}(0)+\int_{0}^{T} u^{\prime \prime}(t) v^{\prime \prime}(t) d t, \quad u, v \in W[0, T], \\
\|u\|_{w}=\sqrt{\langle u, u\rangle_{W}}, \quad u \in W[0, T] .
\end{gathered}
$$

Remark 2.2. The space $W[0, T]$ is a reproducing kernel space, and its reproducing kernel is given by

$$
K_{s}(t)= \begin{cases}s t+\frac{s}{2} t^{2}-\frac{1}{6} t^{3} & t \leq s, \\ s t+\frac{s^{2}}{2} t-\frac{1}{6} s^{3} & t>s .\end{cases}
$$

Definition 2.3. $\widetilde{W}[0, T]=\left\{u(t) \mid u(t)\right.$ is absolutely continuous in $\left.[0, T], u^{\prime}(t) \in L^{2}[0, T]\right\}$. The inner product and norm in $\widetilde{W}[0, T]$ are defined, respectively, by

$$
\begin{gathered}
\langle u, v\rangle_{\widetilde{W}}=u(0) v(0)+\int_{0}^{T} u^{\prime}(t) v^{\prime}(t) d t, \quad u, v \in \widetilde{W}[0, T] \\
\|u\|_{\widetilde{W}}=\sqrt{\langle u, u\rangle_{\widetilde{W}}}, \quad u \in \widetilde{W}[0, T]
\end{gathered}
$$

Remark 2.4. The space $\widetilde{W}[0, T]$ is a reproducing kernel space and its reproducing kernel is given by

$$
G_{s}(t)= \begin{cases}1+t & t \leq s \\ 1+s & t>s .\end{cases}
$$

Remark 2.5. The definition of the spaces $W[0, T]$ and $\widetilde{W}[0, T]$ is suitable for our numerical examples and in general $W[0, T]$ is defined on the basis of the operator $L$, and $\widetilde{W}[0, T]$ must be defined based on the operator $N$.

In order to represent the analytical solution of the model problem, we can assume that $L: W[0, T] \rightarrow \widetilde{W}[0, T]$ is an invertible bounded linear operator, choose a countable dense subset $\left\{t_{i}\right\}_{i=1}^{\infty}$ in $[0, T]$ and define

$$
\phi_{i}(t)=G_{t_{i}}(t), \quad \psi_{\mathrm{i}}(t)=L^{*} \phi_{i}(t),
$$


where $L^{*}$ is the adjoint operator of $L$. The orthonormal system $\left\{\bar{\psi}_{i}(t)\right\}_{i=1}^{\infty}$ of $W[0, T]$ can be derived from the Gram-Schmidt orthogonalization process of $\left\{\psi_{i}(t)\right\}_{i=1}^{\infty}$ as

$$
\bar{\psi}_{i}(t)=\sum_{k=1}^{i} \beta_{i k} \psi_{k}(t)
$$

Theorem 2.6. Suppose that $\left\{t_{i}\right\}_{i=1}^{\infty}$ is dense in $[0, T]$, then $\left\{\psi_{i}(t)\right\}_{i=1}^{\infty}$ is a complete system in $W[0, T]$ and $\psi_{i}(t)=\left.L_{s} K_{s}(t)\right|_{s=t_{i}}$.

Proof. See [22].

Theorem 2.7. If $\left\{t_{i}\right\}_{i=1}^{\infty}$ is dense in $[0, T]$, then the analytical solution of (2.1)-(2.2) will be

$$
u_{n}(t)=\sum_{i=1}^{\infty} \sum_{k=1}^{i} \beta_{i k} N\left(t_{k}, u_{n+p_{1}}\left(t_{k}\right), \ldots, u_{n+p_{d}}\left(t_{k}\right)\right) \bar{\psi}_{i}(t)
$$

Proof. Since $\left\{\bar{\psi}_{i}(t)\right\}_{i=1}^{\infty}$ is a complete system in $W[0, T]$, we have

$$
\begin{aligned}
u_{n}(t) & =\sum_{i=1}^{\infty}\left\langle u_{n}(t), \bar{\psi}_{i}(t)\right\rangle_{W} \bar{\psi}_{i}(t)=\sum_{i=1}^{\infty} \sum_{k=1}^{i} \beta_{i k}\left\langle u_{n}(t), \psi_{k}(t)\right\rangle_{W} \bar{\psi}_{i}(t) \\
& =\sum_{i=1}^{\infty} \sum_{k=1}^{i} \beta_{i k}\left\langle u_{n}(t), L^{*} \phi_{k}(t)\right\rangle_{W} \bar{\psi}_{i}(t)=\sum_{i=1}^{\infty} \sum_{k=1}^{i} \beta_{i k}\left\langle L u_{n}(t), \phi_{k}(t)\right\rangle_{\widetilde{W}} \bar{\psi}_{i}(t) \\
& =\sum_{i=1}^{\infty} \sum_{k=1}^{i} \beta_{i k}\left\langle L u_{n}(t), G_{t_{k}}(t)\right\rangle_{\widetilde{W}} \bar{\psi}_{i}(t)=\sum_{i=1}^{\infty} \sum_{k=1}^{i} \beta_{i k} L u_{n}\left(t_{k}\right) \bar{\psi}_{i}(t) \\
& =\sum_{i=1}^{\infty} \sum_{k=1}^{i} \beta_{i k} N\left(t_{k}, u_{n+p_{1}}\left(t_{k}\right), \ldots, u_{n+p_{d}}\left(t_{k}\right)\right) \bar{\psi}_{i}(t) .
\end{aligned}
$$

Now the approximate solution $u_{n, m}(t)$ can be obtained by the $m$-term intercept of the analytical solution $u_{n}(t)$, that is,

$$
u_{n, m}(t)=\sum_{i=1}^{m} \sum_{k=1}^{i} \beta_{i k} N\left(t_{k}, u_{n+p_{1}}\left(t_{k}\right), \ldots, u_{n+p_{d}}\left(t_{k}\right)\right) \bar{\psi}_{i}(t)
$$

Obviously,

$$
\left\|u_{n, m}(t)-u_{n}(t)\right\|_{W} \longrightarrow 0, \quad(m \longrightarrow \infty)
$$


In the sequel, for evaluating (2.11), we choose nonnegative integer $m$ and put the initial function $u_{n, 0}(t)=0$. Then the approximate solution is defined by

$$
u_{n, m}(t)=\sum_{i=1}^{m} B_{i} \bar{\psi}_{i}(t)
$$

where

$$
B_{i}=\sum_{k=1}^{i} \beta_{i k} N\left(t_{k}, u_{n+p_{1}, k-1}\left(t_{k}\right), \ldots, u_{n+p_{d}, k-1}\left(t_{k}\right)\right) .
$$

Lemma 2.8. If $u_{n, m} \stackrel{\|\cdot\|}{\longrightarrow} \bar{u}_{n},\left\|u_{n, m}\right\|$ is bounded in (2.13), $t_{m} \rightarrow$ s and $N\left(t, u_{n+p_{1}}(t), \ldots, u_{n+p_{d}}(t)\right)$ is continuous, then $N\left(t_{m}, u_{n+p_{1}, m-1}\left(t_{m}\right), \ldots, u_{n+p_{d}, m-1}\left(t_{m}\right)\right) \rightarrow N\left(s, \bar{u}_{n+p_{1}}(s), \ldots, \bar{u}_{n+p_{d}}(s)\right)$.

Proof. Note that

$$
\begin{aligned}
&\left|u_{n+p_{i}}(t)\right|=\left|\left\langle u_{n+p_{i}}(s), K_{t}(s)\right\rangle_{W}\right| \leq\left\|u_{n+p_{i}}(s)\right\|_{W}\left\|K_{t}(s)\right\|_{W} \leq c_{0}\left\|u_{n+p_{i}}(s)\right\|_{W^{\prime}} \\
&\left|\frac{\partial u_{n+p_{i}}(t)}{\partial t}\right|=\left|\left\langle u_{n+p_{i}}(s), \frac{\partial K_{t}(s)}{\partial t}\right\rangle_{W}\right| \leq\left\|u_{n+p_{i}}(s)\right\|_{W}\left\|\frac{\partial K_{t}(s)}{\partial t}\right\|_{W} \leq c_{1}\left\|u_{n+p_{i}}(s)\right\|_{W^{\prime}} \\
&\left|u_{n+p_{i}, m-1}(s)-\bar{u}_{n+p_{i}}(s)\right|=\left|\left\langle u_{n+p_{i}, m-1}(t)-\bar{u}_{n+p_{i}}(t), K_{S}(t)\right\rangle_{W}\right| \\
& \leq\left\|u_{n+p_{i}, m-1}(t)-\bar{u}_{n+p_{i}}(t)\right\|_{W}\left\|K_{t}(s)\right\|_{W} \\
& \leq c_{0}\left\|u_{n+p_{i}, m-1}(t)-\bar{u}_{n+p_{i}}(t)\right\|_{W} .
\end{aligned}
$$

On the other hand, we have

$$
\begin{aligned}
\left|u_{n+p_{i}, m-1}\left(t_{m}\right)-\bar{u}_{n+p_{i}}(s)\right| & =\left|u_{n+p_{i}, m-1}\left(t_{m}\right)-u_{n+p_{i}, m-1}(s)+u_{n+p_{i}, m-1}(s)-\bar{u}_{n+p_{i}}(s)\right| \\
& \leq\left|\partial_{t} u_{n+p_{i}, m-1}(\eta)\right|\left|t_{m}-s\right|+\left|u_{n+p_{i}, m-1}(s)-\bar{u}_{n+p_{i}}(s)\right| .
\end{aligned}
$$

From $u_{n, m} \stackrel{\|\cdot\|}{\longrightarrow} \bar{u}_{n}$ and former statements, it follows that

$$
\left|u_{n+p_{i}, m-1}(s)-\bar{u}_{n+p_{i}}(s)\right| \longrightarrow 0, \quad\left|\partial_{t} u_{n+p_{i}, m-1}(\eta)\right| \leq c_{1}\left\|u_{n+p_{i}, m-1}\right\|_{w} .
$$

Therefore,

$$
\left|u_{n+p_{i}, m-1}\left(t_{m}\right)-\bar{u}_{n+p_{i}}(s)\right| \longrightarrow 0, \quad \text { as } m \longrightarrow \infty,
$$


because of the boundedness of $\left\|u_{n, m}\right\|$. The continuity of $N\left(t, u_{n+p_{1}}(t), \ldots, u_{n+p_{d}}(t)\right)$ implies that

$$
N\left(t_{m}, u_{n+p_{1}, m-1}\left(t_{m}\right), \ldots, u_{n+p_{d}, m-1}\left(t_{m}\right)\right) \longrightarrow N\left(s, \bar{u}_{n+p_{1}}(s), \ldots, \bar{u}_{n+p_{d}}(s)\right), \quad \text { as } m \longrightarrow \infty
$$

Theorem 2.9. Suppose that $\left\|u_{n, m}\right\|$ is bounded in (2.13), and (2.1)-(2.2) has a unique solution. If $\left\{t_{i}\right\}_{i=1}^{\infty}$ is dense in $[0, T]$, then the $m$-term approximate solution $u_{n, m}(t)$ derived from the above method converges to the analytical solution $u_{n}(t)$ of (2.1)-(2.2) and

$$
u_{n}(t)=\sum_{i=1}^{\infty} B_{i} \bar{\psi}_{i}(t)
$$

where $B_{i}$ is given by (2.14).

Proof. At first, we prove the convergence of $u_{n, m}(t)$. From (2.13), we infer that

$$
u_{n, m+1}(t)=u_{n, m}(t)+B_{m+1} \bar{\psi}_{m+1}(t) .
$$

The orthonormality of $\left\{\bar{\psi}_{i}\right\}_{i=1}^{\infty}$ yields that

$$
\left\|u_{n, m+1}\right\|^{2}=\left\|u_{n, m}\right\|^{2}+B_{m+1}^{2}=\cdots=\sum_{i=1}^{m+1} B_{i}^{2} .
$$

In terms of (2.22), it holds that $\left\|u_{n, m+1}\right\| \geq\left\|u_{n, m}\right\|$. Due to the condition that $\left\|u_{n, m}\right\|$ is bounded, $\left\|u_{n, m}\right\|$ is convergent and there exists a constant $c$ such that $\sum_{i=1}^{\infty} B_{i}^{2}=c$. If $l>m$, and then

$$
\begin{aligned}
\left\|u_{n, l}-u_{n, m}\right\|^{2} & =\left\|u_{n, l}-u_{n, l-1}+u_{n, l-1}-u_{n, l-2}+\cdots+u_{n, m+1}-u_{n, m}\right\|^{2} \\
& =\left\|u_{n, l}-u_{n, l-1}\right\|^{2}+\left\|u_{n, l-1}-u_{n, l-2}\right\|^{2}+\cdots+\left\|u_{n, m+1}-u_{n, m}\right\|^{2} .
\end{aligned}
$$

On account of $\left\|u_{n, l}-u_{n, l-1}\right\|^{2}=B_{l}^{2}$, consequently, we have

$$
\left\|u_{n, l}-u_{n, m}\right\|^{2}=\sum_{i=m+1}^{l} B_{i}^{2} \longrightarrow 0 \quad \text { as } m \longrightarrow \infty
$$

The completeness of $W[0, T]$ shows that $u_{n, m} \rightarrow \bar{u}_{n}$ as $m \rightarrow \infty$. Now, we prove that $\bar{u}_{n}$ is the solution of (2.1)-(2.2). Taking limits in (2.13), we get

$$
\bar{u}_{n}(t)=\sum_{i=1}^{\infty} B_{i} \bar{\psi}_{i}(t)
$$


Note that

$$
\begin{aligned}
\left(L \bar{u}_{n}\right)(t) & =\sum_{i=1}^{\infty} B_{i} L \bar{\psi}_{i}(t) \\
\left(L \bar{u}_{n}\right)\left(t_{l}\right) & =\sum_{i=1}^{\infty} B_{i} L \bar{\psi}_{i}\left(t_{l}\right)=\sum_{i=1}^{\infty} B_{i}\left\langle L \bar{\psi}_{i}(t), \phi_{l}(t)\right\rangle_{\widetilde{W}} \\
& =\sum_{i=1}^{\infty} B_{i}\left\langle\bar{\psi}_{i}(t), L^{*} \phi_{l}(t)\right\rangle_{W}=\sum_{i=1}^{\infty} B_{i}\left\langle\bar{\psi}_{i}(t), \psi_{l}(t)\right\rangle_{W} .
\end{aligned}
$$

Therefore, consider

$$
\sum_{l=1}^{i} \beta_{i l}\left(L \bar{u}_{n}\right)\left(t_{l}\right)=\sum_{i=1}^{\infty} B_{i}\left\langle\bar{\psi}_{i}(t), \sum_{l=1}^{i} \beta_{i l} \psi_{l}(t)\right\rangle_{W}=\sum_{i=1}^{\infty} B_{i}\left\langle\bar{\psi}_{i}(t), \bar{\psi}_{l}(t)\right\rangle_{W}=B_{l} .
$$

In view of (2.14), we have

$$
L \bar{u}_{n}\left(t_{l}\right)=N\left(t_{l}, u_{n+p_{1}, l-1}\left(t_{l}\right), \ldots, u_{n+p_{d}, l-1}\left(t_{l}\right)\right) .
$$

Since $\left\{t_{i}\right\}_{i=1}^{\infty}$ is dense in $[0, T]$, for each $s \in[0, T]$, there exist a subsequence $\left\{t_{m_{j}}\right\}_{j=1}^{\infty}$ such that

$$
t_{m_{j}} \rightarrow s \quad(j \longrightarrow \infty)
$$

We have known that

$$
L \bar{u}_{n}\left(t_{m_{j}}\right)=N\left(t_{m_{j}}, u_{n+p_{1}, m_{j}-1}\left(t_{m_{j}}\right), \ldots, u_{n+p_{d}, m_{j}-1}\left(t_{m_{j}}\right)\right) .
$$

Let $j \rightarrow \infty$, by Lemma 2.8 and the continuity of $N$, we have

$$
\left(L \bar{u}_{n}\right)(s)=N\left(s, \bar{u}_{n+p_{1}}(s), \ldots, \bar{u}_{n+p_{d}}(s)\right),
$$

which indicates that $\bar{u}_{n}$ satisfies (2.1)-(2.2).

\section{Numerical Results}

To test the accuracy of the proposed method, three examples are treated in this section. The results are compared with the exact solutions. All experiments are done by taking $T=1$.

Example 3.1. Consider the Volterra equation of the form

$$
\frac{\partial u_{n}}{\partial t}=u_{n}\left(u_{n-1}-u_{n+1}\right)
$$


Table 1: Relative errors of $u_{4, m}(t)$, for Example 3.1.

\begin{tabular}{lcccc}
\hline$t$ & $u_{\text {exact }}$ & $\mathrm{RE}(m=16)$ & $\mathrm{RE}(m=50)$ & $\mathrm{RE}(m=150)$ \\
\hline 0.1 & 3.33333 & $2.39250 E-02$ & $2.15212 E-03$ & $2.27949 E-04$ \\
0.2 & 2.85714 & $1.81724 E-02$ & $1.63061 E-03$ & $1.72270 E-04$ \\
0.3 & 2.50000 & $1.47060 E-02$ & $1.28701 E-03$ & $1.35604 E-04$ \\
0.4 & 2.22222 & $1.21036 E-02$ & $1.04777 E-03$ & $1.10095 E-04$ \\
0.5 & 2.00000 & $1.02211 E-02$ & $8.73935 E-04$ & $9.15787 E-05$ \\
0.6 & 1.81818 & $8.78692 E-03$ & $7.43272 E-04$ & $7.76766 E-05$ \\
0.7 & 1.66667 & $7.65804 E-03$ & $6.42308 E-04$ & $6.69478 E-05$ \\
0.8 & 1.53846 & $6.77754 E-03$ & $5.62483 E-04$ & $5.84769 E-05$ \\
0.9 & 1.42857 & $6.04603 E-03$ & $4.98142 E-04$ & $5.16585 E-05$ \\
1 & 1.33333 & $5.45125 E-03$ & $4.45418 E-04$ & $4.60794 E-05$ \\
\hline
\end{tabular}

With the initial condition $u_{n}(0)=n$, it is easy to check that the exact solution will be

$$
u_{n}(t)=\frac{n}{1+2 t}
$$

Taking $n=4$, relative error (RE) of numerical results for $m=16, m=50$, and $m=150$ are reported in Table 1. Accuracy of approximate solutions is getting better as $m$ increases.

Example 3.2. Consider the discrete $\mathrm{mKdV}$ equation

$$
\frac{\partial u_{n}}{\partial t}=\left(\alpha-u_{n}^{2}\right)\left(u_{n+1}-u_{n-1}\right)
$$

With the initial condition $u_{n}(0)=\sqrt{-\alpha} \tanh (k / 2)(1+\cosh (k)) \operatorname{sech}(k n-2)$, where $k$ and $\alpha$ are arbitrary constants, the exact solution will be

$$
u_{n}(t)=\sqrt{-\alpha} \tanh \left(\frac{k}{2}\right)(1+\cosh (k)) \operatorname{sech}\left(k n+2 \alpha \tanh \left(\frac{k}{2}\right)(1+\cosh (k)) t-2\right) .
$$

Taking $m=50, \alpha=-0.1$ and $k=0.3$ and choosing $n=10$ and $n=15$, we have listed relative error of approximate solutions at some nodal points in Table 2. Numerical results are in good agreement with the exact solutions.

Example 3.3. Consider the discrete sine-Gordon equation of the form

$$
\frac{\partial u_{n+1}}{\partial t}-\frac{\partial u_{n}}{\partial t}=\sin \left(u_{n+1}-u_{n}\right)
$$

With the initial condition $u_{n}(0)=2 \arctan (\tanh (k n+\delta))$ where $\delta$ and $k \neq 0$ are arbitrary constants, the exact solution will be

$$
u_{n}(t)=2 \arctan \left(\tanh \left(k n-\frac{1}{2 \tanh (k)} t+\delta\right)\right) .
$$


Table 2: Relative errors of $u_{n, 50}(t)$, for Example 3.2.

\begin{tabular}{lcc}
\hline$t$ & $\mathrm{RE}(n=10)$ & $\mathrm{RE}(n=15)$ \\
\hline 0.1 & $9.28456 E-06$ & $1.31645 E-05$ \\
0.2 & $4.00254 E-06$ & $3.45703 E-06$ \\
0.3 & $7.57456 E-06$ & $3.88922 E-05$ \\
0.4 & $2.41475 E-05$ & $8.78319 E-05$ \\
0.5 & $4.59293 E-05$ & $1.49880 E-04$ \\
0.6 & $7.31335 E-05$ & $2.24650 E-04$ \\
0.7 & $1.05974 E-04$ & $3.11759 E-04$ \\
0.8 & $1.44666 E-04$ & $4.10836 E-04$ \\
0.9 & $1.89424 E-04$ & $5.21514 E-04$ \\
1 & $2.40464 E-04$ & $6.43435 E-04$ \\
\hline
\end{tabular}

Table 3: Relative errors of $u_{n, 50}(t)$, for Example 3.3.

\begin{tabular}{lcc}
\hline$t$ & $\mathrm{RE}(n=5)$ & $\mathrm{RE}(n=10)$ \\
\hline 0.1 & $1.36553 E-10$ & $6.79847 E-12$ \\
0.2 & $2.58908 E-08$ & $6.18223 E-08$ \\
0.3 & $1.51271 E-07$ & $3.55847 E-07$ \\
0.4 & $5.55149 E-07$ & $1.27165 E-06$ \\
0.5 & $1.79618 E-06$ & $3.99090 E-06$ \\
0.6 & $5.56118 E-06$ & $1.19763 E-05$ \\
0.7 & $1.69167 E-05$ & $3.53422 E-05$ \\
0.8 & $5.10327 E-05$ & $1.03601 E-04$ \\
0.9 & $1.53212 E-04$ & $3.02802 E-04$ \\
1 & $4.58423 E-04$ & $8.83675 E-04$ \\
\hline
\end{tabular}

Taking $m=50, \delta=9$ and $k=0.3$ and choosing $n=5$ and $n=10$, we have given relative error of approximate solutions at some nodal points in Table 3. Numerical results are in good agreement with the exact solutions.

\section{Conclusion}

In this work, we proposed an algorithm for solving a class of nonlinear differential-difference equations on the basis of reproducing kernel spaces. Results of numerical examples show that the present method is an accurate and reliable analytical-numerical technique for solving such differential-difference equations. The method is shown to be of good convergence, simple in principle, and easy to program.

\section{References}

[1] M. Toda, Theory of Nonlinear Lattices, vol. 20 of Springer Series in Solid-State Sciences, Springer, Berlin, Germany, 1981.

[2] G.-C. Wu, L. Zhao, and J.-H. He, "Differential-difference model for textile engineering," Chaos, Solitons and Fractals, vol. 42, no. 1, pp. 352-354, 2009.

[3] J.-H. He and E. W. M. Lee, "Variational principle for the differential-difference system arising in stratified hydrostatic flows," Physics Letters Section A, vol. 373, no. 18-19, pp. 1644-1645, 2009. 
[4] S.-D. Zhu, "Discrete $(2+1)$-dimensional Toda lattice equation via Exp-function method," Physics Letters A, vol. 372, no. 5, pp. 654-657, 2008.

[5] R. Mokhtari, "Variational iteration method for solving nonlinear differential-difference equations," International Journal of Nonlinear Sciences and Numerical Simulation, vol. 9, no. 1, pp. 19-23, 2008.

[6] X.-M. Qian, S. Y. Lou, and X.-B. Hu, “Variable separation approach for a differential-difference system: special Toda equation," Journal of Physics A, vol. 37, no. 6, pp. 2401-2411, 2004.

[7] D. Baldwin, Ü. Göktaş, and W. Hereman, "Symbolic computation of hyperbolic tangent solutions for nonlinear differential-difference equations," Computer Physics Communications, vol. 162, no. 3, pp. 203-217, 2004.

[8] L. Wu, L.-D. Xie, and J.-F. Zhang, "Adomian decomposition method for nonlinear differentialdifference equations," Communications in Nonlinear Science and Numerical Simulation, vol. 14, no. 1 , pp. 12-18, 2009.

[9] Z. Wang, L. Zou, and Z. Zong, "Adomian decomposition and Padé approximate for solving differential-difference equation," Applied Mathematics and Computation, vol. 218, no. 4, pp. 1371-1378, 2011.

[10] A. Yildirim, "Exact solutions of nonlinear differential-difference equations by he's homotopy perturbation method," International Journal of Nonlinear Sciences and Numerical Simulation, vol. 9, no. 2, pp. 111-114, 2008.

[11] A. Yildirim, "He's homotopy perturbation method for nonlinear differential-difference equations," International Journal of Computer Mathematics, vol. 87, no. 5, pp. 992-996, 2010.

[12] M. A. Abdou, "New applications of He's homotopy perturbation method for nonlinear differential difference equations," Physica Scripta, vol. 81, no. 1, Article ID 015003, 2010.

[13] M. M. Mousa and A. Kaltayev, "Homotopy perturbation method for solving nonlinear differentialdifference equations," Zeitschrift fur Naturforschung Section A, vol. 65, no. 6-7, pp. 511-517, 2010.

[14] M. M. Mousa, A. Kaltayev, and H. Bulut, "Extension of the homotopy perturbation method for solving nonlinear differential-difference equations," Zeitschrift fur Naturforschung Section A, vol. 65, no. 12, pp. 1060-1064, 2010.

[15] N. Aronszajn, "Theory of reproducing kernels," Transactions of the American Mathematical Society, vol. 68, pp. 337-404, 1950.

[16] P. Bouboulis and M. Mavroforakis, "Reproducing kernel Hilbert spaces and fractal interpolation," Journal of Computational and Applied Mathematics, vol. 235, no. 12, pp. 3425-3434, 2011.

[17] F. Geng, "Solving singular second order three-point boundary value problems using reproducing kernel Hilbert space method," Applied Mathematics and Computation, vol. 215, no. 6, pp. 2095-2102, 2009.

[18] F. Geng, "A novel method for solving a class of singularly perturbed boundary value problems based on reproducing kernel method," Applied Mathematics and Computation, vol. 218, no. 8, pp. 4211-4215, 2011.

[19] F. Geng and M. Cui, "A reproducing kernel method for solving nonlocal fractional boundary value problems," Applied Mathematics Letters, vol. 25, no. 5, pp. 818-823, 2012.

[20] F. Z. Geng and X. M. Li, "A new method for Riccati differential equations based on reproducing kernel and quasilinearization methods," Abstract and Applied Analysis, vol. 2012, Article ID 603748, 8 pages, 2012.

[21] M. Cui and F. Geng, "A computational method for solving one-dimensional variable-coefficient Burgers equation," Applied Mathematics and Computation, vol. 188, no. 2, pp. 1389-1401, 2007.

[22] M. Mohammadi and R. Mokhtari, "Solving the generalized regularized long wave equation on the basis of a reproducing kernel space," Journal of Computational and Applied Mathematics, vol. 235, no. 14, pp. 4003-4014, 2011.

[23] M. Cui and Y. Lin, Nonlinear Numerical Analysis in the Reproducing Kernel Space, Nova Science, New York, NY, USA, 2009. 


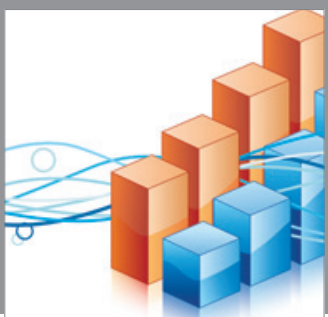

Advances in

Operations Research

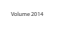

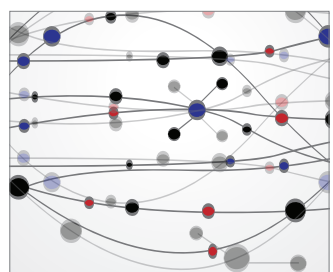

\section{The Scientific} World Journal
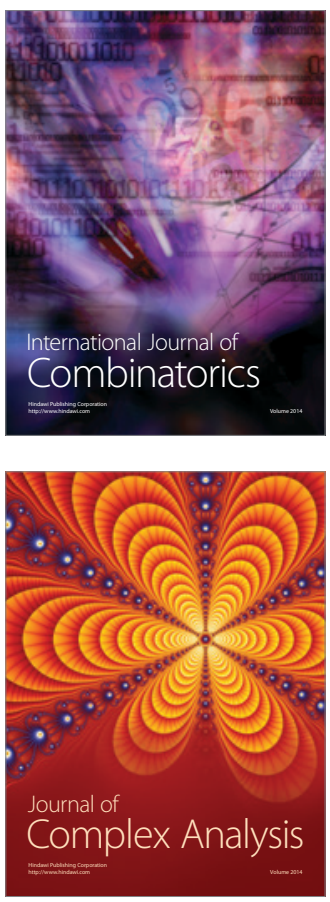

International Journal of

Mathematics and

Mathematical

Sciences
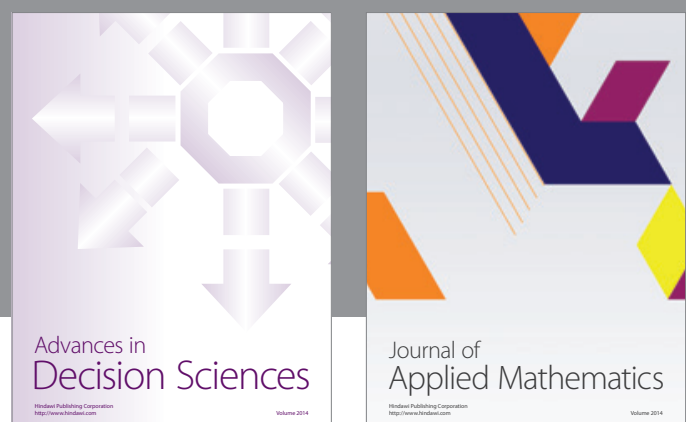

Journal of

Applied Mathematics
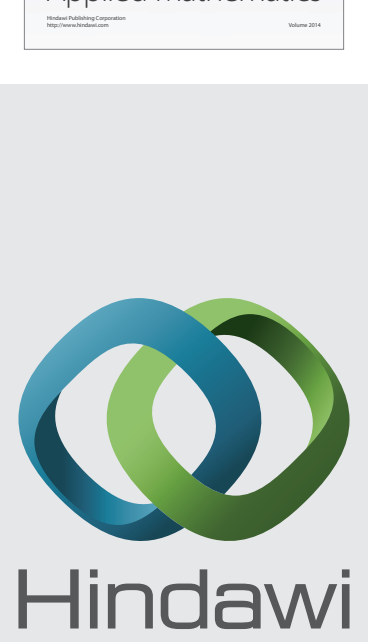

Submit your manuscripts at http://www.hindawi.com
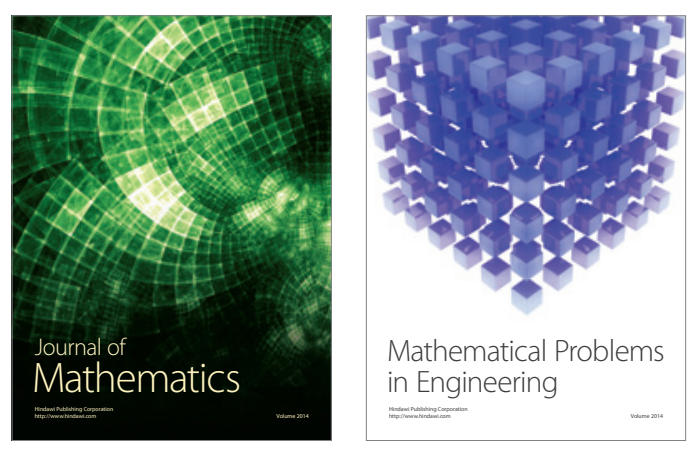

Mathematical Problems in Engineering
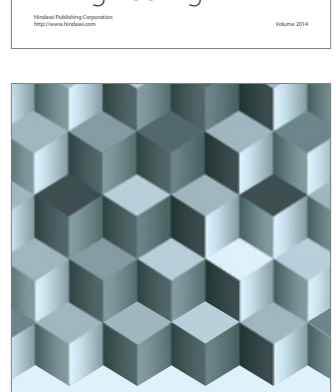

Journal of

Function Spaces
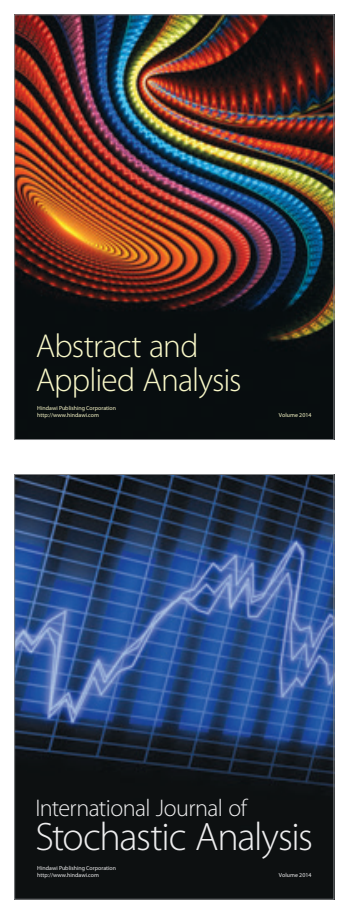

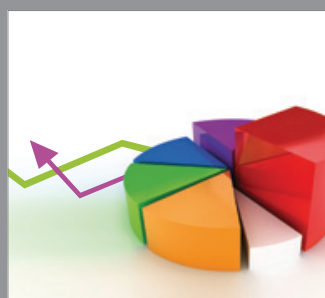

ournal of

Probability and Statistics

Promensencen
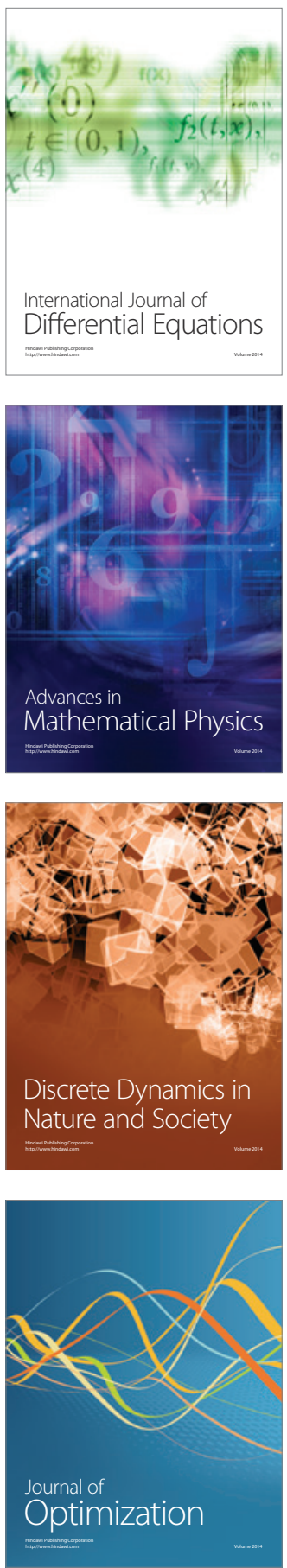